\title{
X-ray photoemission and absorption spectroscopy of supported nanoscale iron clusters
}

\author{
K. W. Edmonds, S. H. Baker, S. C. Thornton, M. J. Maher, A. M. Keen, ${ }^{a}$ and C. Binns ${ }^{\text {b) }}$ \\ Department of Physics and Astronomy, University of Leicester, Leicester LE1 7RH, United Kingdom
}

(Received 5 April 1999; accepted for publication 5 April 1999)

\begin{abstract}
Fe particles with sizes in the range $1-5 \mathrm{~nm}$, formed by a gas-aggregation method and deposited onto graphite and $\mathrm{C}_{60}$ supports, were studied by $\mathrm{x}$-ray photoemission spectroscopy, $\mathrm{x}$-ray absorption spectroscopy, and magnetic linear dichroism. Clusters deposited onto a $\mathrm{C}_{60}$ coated graphite substrate become embedded within the fullerene film, and have an increased resistance to oxidation compared to exposed clusters supported on a graphite surface. No evidence for hybridization between the electronic states of $\mathrm{Fe}$ and $\mathrm{C}_{60}$ is seen. The magnetic dichroism signal of the exposed clusters increases sharply with the film thickness because of the increased cluster interactions. (C) 1999 American Institute of Physics. [S0021-8979(99)05717-5]
\end{abstract}

\section{INTRODUCTION}

Nanoscale particles define a boundary between the atomic and the solid state. Novel electronic and magnetic properties can occur in such systems, arising from the high proportion of low coordinated atoms at the surface, a modified atomic arrangement, the quantum size effect, and the confinement of the Fermi sea to a small volume. Previous studies have shown that such systems can possess magnetic moments that are enhanced with respect to the bulk. ${ }^{1-3}$

In x-ray photoemission spectroscopy (XPS), electrons are excited from bound states to continuum states far above the Fermi level $E_{F}$. This is a popular tool for the study of small metal particles. ${ }^{4-11}$ The perturbed electronic structure in the initial state, ${ }^{4}$ and the reduced screening of the core hole in the final state, ${ }^{5}$ can have considerable influence on the shape and position of the characteristic photoemission peaks of an assembly of nanoscale particles. XPS can also be used to yield magnetic information, in certain experimental arrangements where the photoemission spectrum is a function of the orientation of the sample magnetization $\mathbf{M}$. One of the most versatile of these experiments is magnetic linear dichroism in the angular distribution of photoelectrons (MLDAD). ${ }^{12}$ In MLDAD, photoelectrons are excited with light of linear polarization $\mathbf{P}$, and collected along the direction $\epsilon$. The vectors $\mathbf{M}, \mathbf{P}$, and $\epsilon$ define a chiral experimental geometry. A large change in the photoemission spectrum of core levels possessing an angular momentum quantum number $l>0$, of up to around $40 \%$ of the total intensity, can be observed upon reversing the chirality, i.e., upon reversing $\mathbf{M}$. MLDAD is therefore an element specific probe of surface magnetism. $^{13}$

Another useful probe of electronic structure is X-ray absorption spectroscopy (XAS). Unlike XPS, XAS studies transitions to allowed states just above $E_{F}$. The spectrum

\footnotetext{
a) Also with: Research Institute For Materials, Toernooiveld 1, 6525 ED Nijmegen, The Netherlands.

b) Author to whom correspondence should be addressed; electronic mail: cb12@le.ac.uk
}

therefore yields information concerning the unoccupied part of the local density of states. ${ }^{14}$ In particular, the total intensity associated with an absorption edge is proportional to the number of dipole allowed empty states per atom. When performed with circularly polarized light, XAS can also yield important magnetic information, as $\mathrm{X}$-ray magnetic circular dichroism. $3,14,15$

In the present work, XPS, MLDAD, and XAS were used to characterize Fe clusters deposited onto two substrates: freshly cleaved highly oriented pyrolytic graphite (HOPG), and HOPG coated with a thick film of $\mathrm{C}_{60}$. Both of these are relatively chemically inert, and so the properties of the clusters are only weakly perturbed by the support.

\section{EXPERIMENT}

The portable gas-aggregation source used in this experiment ${ }^{16}$ can deposit a beam of Fe clusters in situ onto substrates within an ultrahigh vacuum (UHV) system. Clusters can be size selected before deposition using a high mass quadrupole filter; or alternatively, a high flux of unfiltered clusters can be deposited. Electrostatic deflectors allow the neutral unfiltered and the ionized size-selected cluster beams to be separated. The neutral clusters land with thermal energies, while the ionized clusters are accelerated to around 20 $\mathrm{eV}$ per cluster before deposition. ${ }^{16}$ The free jet expansion in the cluster source is too weak to cause significant acceleration of the clusters.

Scanning tunnelling microscopy of clusters deposited from this source onto a range of substrates ${ }^{17,18}$ has shown that the unfiltered beam has an asymmetric log-normal size distribution with the peak at $2.3 \mathrm{~nm}(\approx 500$ atoms $)$ and a median diameter of $3.1 \mathrm{~nm}$. No evidence for migration, fragmentation, or coalescence of clusters was observed in these studies, for either the size-selected or the unfiltered depositions. The number of ionized clusters deposited can be accurately measured, by integration of the sample drain current during the deposition. For the neutral clusters, the film thickness was calibrated using a quartz oscillator. In the following, the film thickness is expressed as a fraction of complete monolayers (MLs) of closely packed $2.3 \mathrm{~nm}$ diameter 
clusters. The experiments were performed on Beamlines $5 \mathrm{U}$ and 1.1 of the SRS, Daresbury laboratory. HOPG substrates were cleaved in air and attached to a liquid He cryostat within the beamline UHV end stations. The cluster source was coupled to the chambers through a gate valve. Before cluster deposition, the substrates were heated to $520 \mathrm{~K}$, which is sufficient to remove measurable contaminants from the surface.

Clusters were deposited with the substrate cooled to $30 \mathrm{~K}$. During deposition, the chamber pressure was $\approx 10^{-5}$ mbar due to the presence of the He bath gas used in the cluster formation, while the partial pressure of the residual contaminants remained at the $10^{-11}-10^{-12} \mathrm{mbar}$ level. After the gate valve was closed, the vacuum recovered to better than $10^{-10} \mathrm{mbar}$ within $2 \mathrm{~min}$. Some of the substrates were covered with a thick layer of $\mathrm{C}_{60}$ prior to cluster deposition, by the sublimation of $99.5 \%$ pure $\mathrm{C}_{60}$ powder within the UHV chamber. A "conventional" Knudsen cell type UHV Fe evaporator was also used to produce Fe films during the experiments.

XPS measurements were performed using a HA100 CHA with multichannel detection. Photoelectrons were collected at $40^{\circ}$ to the photon beam. For the MLDAD measurements, spectra were taken after magnetic field pulses were applied along the sample surface, and perpendicular to the plane defined by $\mathbf{P}$ and $\epsilon$, using $\mathrm{Cu}$ coils. XAS measurements were performed by measuring the sample drain current as the photon energy was swept through the $\mathrm{Fe} L_{2,3}$ edges.

\section{RESULTS AND DISCUSSION}

Two samples consisting of $0.02 \mathrm{ML}$ of Fe clusters mass selected in the size range $2.5-3.5 \mathrm{~nm}$ were produced, the first by deposition directly onto HOPG, and the second by deposition on a HOPG substrate coated with an $80 \AA$ thick layer of $\mathrm{C}_{60}$. The XAS Fe $L_{3,2}$ were measured under the same conditions for each sample. The edge jump, normalized to the beam intensity, was found to be smaller for the $\mathrm{C}_{60}$-covered substrate by a factor 7.5 , even though the amount of Fe within each sample is the same to within 5\%. The likely cause of the reduction in intensity is that the $\mathrm{Fe}$ clusters become embedded within the $\mathrm{C}_{60}$ film. Using a total electron yield sampling depth of $17 \AA$ for the $\mathrm{Fe} L_{3,2}$ edge as given in Ref. 15 , this implies that on average the clusters are buried in the $\mathrm{C}_{60}$ film to a depth of $35 \AA$, or 3-4 molecular layers. This is in contrast to a monolayer of $\mathrm{C}_{60}$ upon $\mathrm{Si}(111)$, where a STM study has shown that deposited metal clusters remain above the $\mathrm{C}_{60}$ layer. ${ }^{17}$

Figure 1 shows the XAS after subtraction of a linear background fit to the pre-edge region, and multiplication of the $\mathrm{Fe} / \mathrm{C}_{60}$ spectrum by 7.5 . The spectral shapes and intensities are indistinguishable within the experimental error. In both cases the spectra have a similar shape and branching ratio (ratio of the $L_{3}$ intensity to the total $L_{3.2}$ intensity) to bulk $\mathrm{Fe},{ }^{14}$ indicating that the clusters do not fragment upon impact. Also, the similarity of the two spectra implies that the band structure for the exposed and the embedded clusters are indistinguishable, with an equal number of $d$-band holes per atom in each case. This indicates that hybridization is

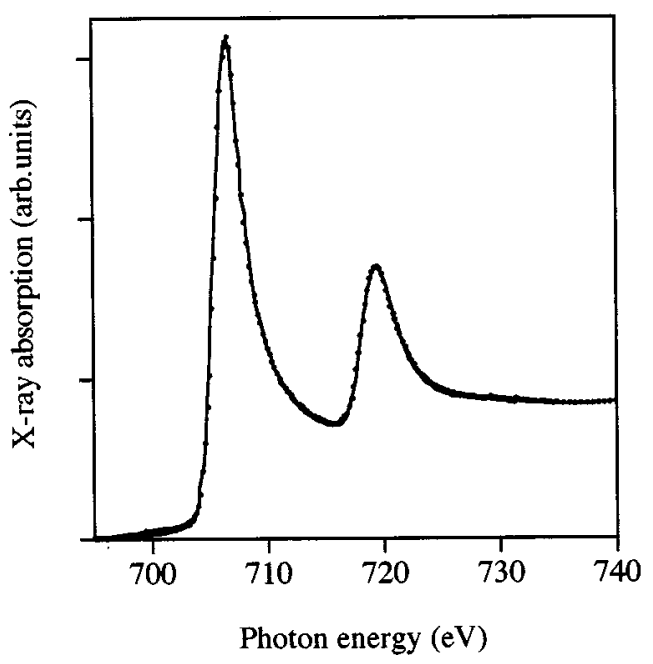

FIG. 1. X-ray absortion spectra for equal coverages of mass-selected Fe clusters on graphite (full line) and on a $\mathrm{C}_{60}$ film (dots), after subtraction of a linear background. The spectrum for $\mathrm{Fe}$ on $\mathrm{C}_{60}$ has been multiplied by a factor of 7.5 .

weak between the electronic levels of $\mathrm{Fe}$ and the $\mathrm{C}_{60}$, in contrast to the results of Ref. 9. The Fe clusters studied in that reference are grown by atomic deposition onto $\mathrm{C}_{60}$, and are smaller than those studied in the present work, which may account for the discrepancy.

Figure 2(a) shows the XPS Fe $3 p$ spectra recorded at a photon energy of $188 \mathrm{eV}$, under the same conditions, for three unmagnetized Fe samples on HOPG: two neutral cluster depositions of density 0.11 and $0.73 \mathrm{ML}$, and a continuous film of thickness $30 \AA$ grown by atomic deposition using the MBE evaporator. The latter spectrum is similar in shape and energy to previously reported bulk-like Fe films on HOPG ${ }^{11}$ There is an increased intensity on the high binding energy side of the peak for the cluster films, which appears to be independent of the film thickness.

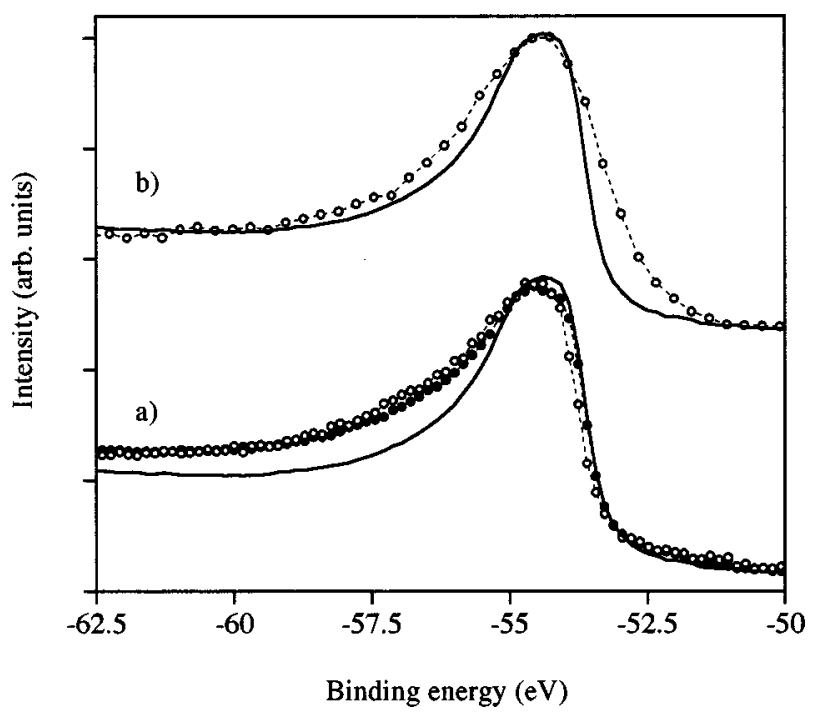

FIG. 2. Comparison of XPS data for a $30 \AA$ continuous film on HOPG (full line), with: (a) 0.11 ML Fe clusters on HOPG (open circles) and 0.73 ML Fe clusters on HOPG (blackcircles); (b) $0.67 \mathrm{ML} \mathrm{Fe}$ clusters on $\mathrm{C}_{60}$ (open circles). 
Figure 2(b) compares the Fe $3 p$ spectra for the continuous film on HOPG with $0.67 \mathrm{ML}$ of unfiltered Fe clusters embedded in $\mathrm{C}_{60}$. The latter is very low in intensity because of the short photoelectron mean free path, and was recorded at a reduced experimental resolution and a higher photon energy $(500 \mathrm{eV})$ than for the Fe/HOPG films. Apart from the Gaussian broadening caused by the lower resolution, the increased intensity tail is not present for the embedded film.

Differences between the photoemission spectra of nanoscale clusters and bulk systems have been measured previously. ${ }^{4-11}$ Typically, a shift to higher binding energies is observed, combined with an increase in width. This may account for some of the intensity of the tail; however, it is surprising that the width hardly changes with increasing coverage, and also that the embedded clusters are not subject to the same broadening as the exposed clusters.

A high intensity tail may be caused by the presence of additional photoemission peaks within the spectrum, corresponding to $\mathrm{Fe}$ in a different chemical state if, for example, the clusters are partially oxidized. Then, the difference in line shape between exposed and embedded clusters is because the $\mathrm{C}_{60}$ overlayers act as a protective coat against oxidation. Therefore, the oxidation occurs after deposition rather than during the cluster formation process.

The spectra were fitted with a line shape matching the $\mathrm{Fe} 3 p$ of the continuous film, plus an additional Lorentzian line shape. The best fit was obtained with the extra peak at around $3 \mathrm{eV}$ higher binding energy than the main peak. This energy shift is close to the shift measured previously for $\mathrm{Fe}^{2+},{ }^{19}$ and indicates the presence of $\mathrm{FeO}$ within the films. A small $\mathrm{O} 1 s$ feature was also observed. The $\mathrm{Fe}^{2+}$ peak accounts for $10 \%-15 \%$ of the total Fe $3 p$ intensity.

No shoulders are visible on the XAS spectra (Fig. 1) for either exposed or embedded clusters, and there is no observable difference in the branching ratio. This may indicate that the oxide is concentrated on the surface of the clusters. At $188 \mathrm{eV}$ photon energy, the photoelectron mean free path is $\approx 5 \AA$, and for a cluster containing six atomic layers, the surface layer will contribute $\approx 42 \%$ of the $\mathrm{Fe} 3 p$ intensity. For total electron yield measurements of the $\mathrm{Fe} L_{3,2}$ edge, the secondary electron attenuation length is $\approx 17 \AA$, and the surface layer contributes only $23 \%$ of the XAS intensity. Therefore the measured $\mathrm{Fe}^{2+}$ contribution to the absorption spectrum is much smaller.

Fe $3 p$ spectra were recorded after applying a magnetic field pulse along two opposite directions. The difference between the spectra $\left(I^{+}-I^{-}\right)$is shown in Fig. 3 for the $30 \AA$ continuous film and for a neutral cluster film of density 1.1 ML on HOPG. The difference spectra are normalized to the same total height of the photoemission spectra $\left(I^{+}+I^{-}\right)$. The MLDAD response of the continuous film is comparable to previous observations for magnetically saturated $\mathrm{Fe}$ films, ${ }^{11-13}$ consisting of \pm intensity lobes on either side of the $3 p$ peak. The maximum asymmetry is $7.5 \%$ on the high binding energy (BE) side, and $-19.7 \%$ on the low BE side. For the cluster film, the dichroism peaks occur at the same energy as for the continuous film, but are reduced in magnitude.

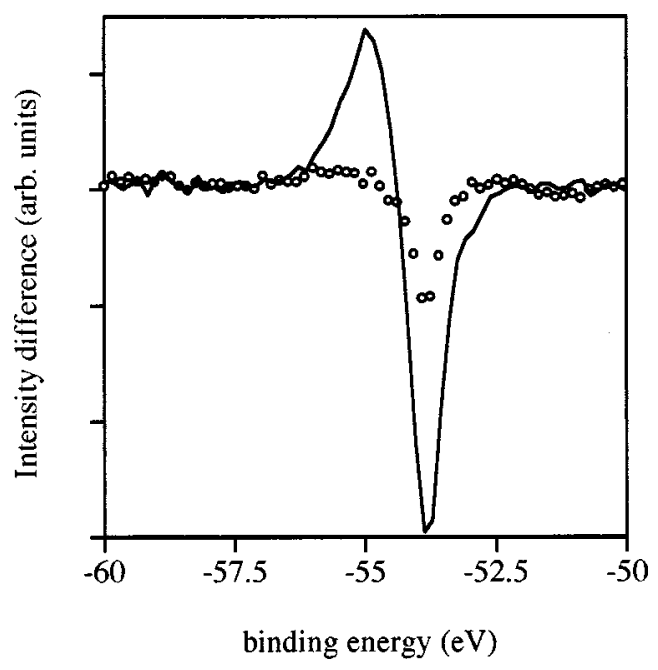

FIG. 3. Difference between XPS spectra taken with opposite sample magnetization directions, for a continuous Fe film on HOPG (full line), and for Fe clusters on HOPG (circles).

MLDAD spectra were recorded for a range of cluster densities. In each case, the asymmetry is confined to the energy range $-57--54.5 \mathrm{eV}$ for the + lobe, and $-54.5-$ $52.5 \mathrm{eV}$ kinetic energy for the - lobe, the same binding energy as for the bulk-like sample. The large intensity at high binding energies shows no asymmetry. This is further evidence that the tail can be attributed to the presence of $\mathrm{FeO}$, as spin-resolved photoemission has shown that the $\mathrm{Fe}^{2+} 3 p$ spectrum is unpolarized. ${ }^{19}$

No dichroism was observed for cluster films deposited on $\mathrm{C}_{60}$. However, calculation of the radial matrix elements for $\mathrm{Fe} 3 p$ photoemission show that the MLDAD signal falls to around $10 \%-15 \%$ of its maximum value for electron kinetic energies of around $450 \mathrm{eV} .{ }^{20}$ Given this and the lower signal-to-noise ratio, the lack of measurable dichroism for the embedded films is unsurprising.

The peak-to-peak asymmetry as a function of cluster density upon HOPG is shown in Fig. 4. The asymmetry is

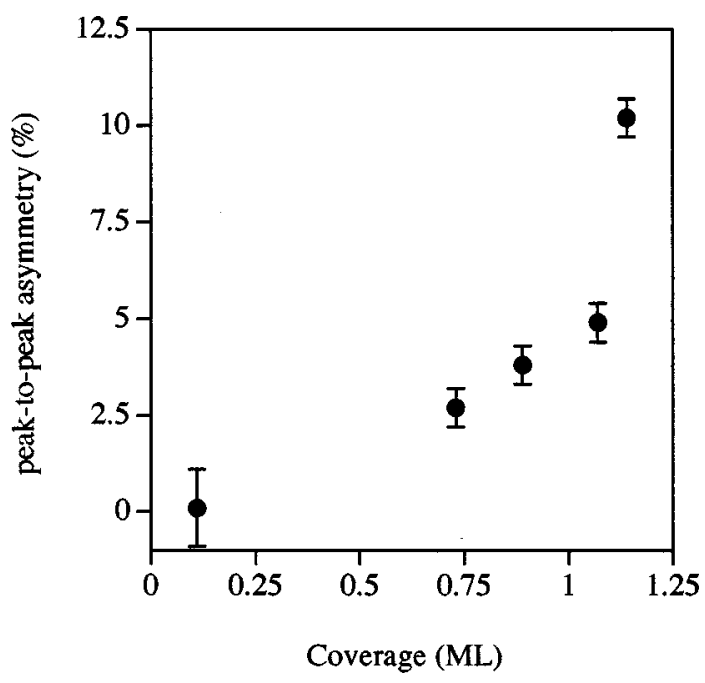

FIG. 4. MLDAD asymmetry versus coverage of Fe clusters on a HOPG surface. 
expressed as a percentage change of the total XPS intensity at a given energy, after background subtraction. The remanent magnetiszation per atom increases sharply with increasing coverage. No dichroism is visible within the noise level for the cluster film of lowest density (0.11 ML).

The lack of dichroism at this low coverage is because of the super paramagnetic nature of the clusters. At a temperature of $30 \mathrm{~K}$ and for particles containing $\sim 500$ atoms, the thermal relaxation of the magnetization is fast compared to the measurement time. ${ }^{21}$ Therefore, there is no detectable remanent magnetization. At higher densities, the interaction between the clusters stabilizes the magnetization at $30 \mathrm{~K}$. Therefore, the dichroism increases rapidly as the coverage approaches a complete layer of clusters.

An increasing remanent magnetization with density for clusters on graphite was also recently observed using magnetic circular dichroism in $\mathrm{x}$-ray absorption. ${ }^{3}$ Unlike the present result, the magnetization was measured out of the film plane. Since the magnetization increases with coverage in both directions, it is apparent that the increasing dichroism with coverage is not due to a switching of the film anisotropy from out-of-plane to in-plane or vice versa. Instead the increase occurs because of the increased resistance of the magnetization of each cluster to thermal fluctuations as intercluster interactions grow stronger. Consistent with this result, an increase in the superparamagnetic blocking temperature with increasing volume filling fraction has been observed for nanoscale Co clusters in $\mathrm{Ag}$ and $\mathrm{SiO}$ matrices. ${ }^{22}$

\section{SUMMARY}

Nanoscale $\mathrm{Fe}$ clusters deposited onto a $\mathrm{C}_{60}$ film on HOPG become embedded within the film. Evidence for oxidation in exposed clusters on HOPG is not present when the clusters are covered by a $\mathrm{C}_{60}$ film. The exposed clusters possess an increasing remanent magnetization with increasing cluster density, because the cluster interactions oppose thermal magnetic relaxation.

${ }^{1}$ J. P. Bucher, D. C. Douglass, and L. A. Bloomfield, Phys. Rev. Lett. 66, 3052 (1991).

${ }^{2}$ I. M. L Billas, A. Châtelain, and W. A. de Heer, Science 265, 1682 (1994).

${ }^{3}$ K. W. Edmonds, C. Binns, S. H. Baker, S. C. Thornton, C. Norris, J. B. Goedkoop, M. Finazzi, and N. B. Brookes, Phys. Rev. B (submitted).

${ }^{4}$ M. G. Mason, Phys. Rev. B 27, 1378 (1983).

${ }^{5}$ G. K. Wertheim, S. B. DiCenzo, and S. E. Youngquist, Phys. Rev. Lett. 51, 2310 (1983).

${ }^{6}$ J. T. P. Cheung et al., Surf. Sci. 140, 151 (1984).

${ }^{7}$ W. Eberhardt, P. Fayet, D. M. Cox, Z. Fu, and A. Kaldor, Phys. Rev. Lett. 64, 780 (1990).

${ }^{8}$ M. W. Ruckman, B. Xia, and D. Shih, Phys. Rev. B 50, 17682 (1994).

${ }^{9}$ M. Biermann, B. Kessler, S. Krummacher, and W. Eberhardt, Solid State Commun. 95, 1 (1995).

${ }^{10}$ A. M. Keen, C. Binns, S. H. Baker, S. Mozley, C. Norris, and S. C. Thornton, Surf. Sci. 352-354, 715 (1996).

${ }^{11}$ S. H. Baker, K. W. Edmonds, C. Binns, A. M. Keen, S. C. Thornton, and C. Norris, Phys. Rev. B (submitted).

${ }^{12}$ Ch. Roth, F. U. Hillebrecht, H. B. Rose, and E. Kisker, Phys. Rev. Lett. 70, 3479 (1993).

${ }^{13}$ F. Sirotti, G. Panaccione, and G. Rossi, Phys. Rev. B 52, 17063 (1995).

${ }^{14}$ F. M. F. De Groot, J. Electron Spectrosc. Relat. Phenom. 67, 529 (1994).

${ }^{15}$ J. Stöhr and R. Nakajima, J. Phys. IV 7, 47 (1997).

${ }^{16}$ S. H. Baker, S. C. Thornton, A. M. Keen, T. I. Preston, C. Norris, K. W. Edmonds, and C. Binns, Rev. Sci. Instrum. 68, 1853 (1997).

${ }^{17}$ M. D. Upward, P. Moriarty, P. H. Beton, S. H. Baker, C. Binns, and K. W. Edmonds, Appl. Phys. Lett. 70, 2114 (1997).

${ }^{18}$ M. D. Upward, Ph.D. Thesis, University of Nottingham, 1998.

${ }^{19}$ B. Sinkovic, P. D. Johnson, N. B. Brookes, A. Clarke, and N. V. Smith, Phys. Rev. Lett. 65, 1647 (1990).

${ }^{20}$ G. van der Laan, Phys. Rev. B 51, 240 (1995).

${ }^{21}$ L. Néel, Ann. Geophys. (C.N.R.S.) 5, 99 (1949).

${ }^{22}$ V. Dupuis, J. Tuaillon, B. Prevel, A. Perez, and P. Melinon, Z. Phys. D 40, 155 (1997). 